\title{
Three Women with Non-Segmental Vitiligo in Three Generations of a Family: A Case Report
}

Fatemeh Chorli $^{1}$, Zahra Ghodsalavi ${ }^{1}$, Maryam Hajilari ${ }^{1}$, Farzad Dastaviz ${ }^{1}$, Majid Mehri ${ }^{2}$, Morteza

\section{Oladnabi*3}

1. Student Research Committee, Golestan University of Medical Sciences, Gorgan, Iran

2. Department of Internal Medicine, School of Medicine, Golestan University of Medical Sciences, Gorgan, Iran

3. Department of Medical Genetics, Gorgan Congenital Malformations Research Center, Golestan University of Medical Sciences, Gorgan, Iran

\section{ABSTRACT}

Background: Vitiligo is a common autoimmune skin disorder, which is characterized by incomplete penetrance and genetic heterogeneity. It is classified into two types: segmental and nonsegmental, and most cases suffer from non-segmental vitiligo. The disease affects about 2-10 in 1000 people in different population with no sex predilection. Furthermore, the genetics of vitiligo cannot be described by Mendelian pattern of inheritance. We herein report three female relatives (niece, aunt, grandmother) with non-segmental vitiligo in a family.

Case description: The symptoms and severity of the disease varied between the cases. Incomplete penetrance was completely evident in this family. The grandmother had not received any treatment, but the aunt and niece were receiving medication and phototherapy. However, these treatments were not beneficial for them.

Conclusion: Currently there is no effective treatment or screening method for vitiligo. Thus, genetic counseling, risk determination and identification of other genetic contributors could be beneficial.

KEYWORDS: Non-segmental vitiligo, Incomplete penetrance, Skin depigmentation

Received: 2018/12/15 Revised: 2019/01/05

Published:2019/01/20

*Correspondence: Morteza Oladnabi

Address: Golestan University of Medical Sciences, Gorgan-Sari Highway, Gorgan, Iran

Telephone: +98-9104495830 Email: oladnabidozin@yahoo.com 


\section{INTRODUCTION}

Vitiligo is a skin disorder characterized by the destruction of melanocytes (1). This chronic idiopathic disorder can be diagnosed by detection of one or more spots with no pigment due to decreased number of melanocytes in skin layers (2). Pathological mechanisms include genetic, neurological, biochemical, viral and autoimmune changes. Recent studies have shown that $1-4 \%$ of the world's population and $1.8 \%$ of Iranians are affected by vitiligo $(3,4)$. The disease often affects the hands, fingers, face and less frequently hair and eyelids. Progressive and rapid depigmentation of different skin areas can be seen in circular, oval and linear shapes (5). Vitiligo is more commonly observed in children and adolescents. Clinical symptoms appear before age of 20 in $50 \%$ of cases, but starts before the age of 14 in $25 \%$ of cases. Due to the changes in the appearance, those affected by the disease suffer from numerous psychological problems including depression, anxiety, paranoia, instability and obsession, all of which significantly reduces quality of life $(6,7)$. These problems lead to serious and permanent consequences in the patients, especially adults $(1,2)$. The skin appearance can determine the patient's physical condition and pathological changes. Depressive episodes are significantly more common in women, especially younger women. Severe stress in married women with progressive vitiligo leads to marital problems, divorce and remarriage. In Iran, patients also suffer from social problems and do not receive enough support from their families.

Genome-wide association studies have discovered approximately 50 different genetic loci that could contribute to development of vitiligo (8). We herein report three female relatives (niece, aunt, grandmother) with nonsegmental vitiligo in a family.

\section{CASE PRESENTATION}

In order to obtain more information on inheritance pattern, a family pedigree was drawn using Cyrillic, a pedigree drawing tool (Figure 1).

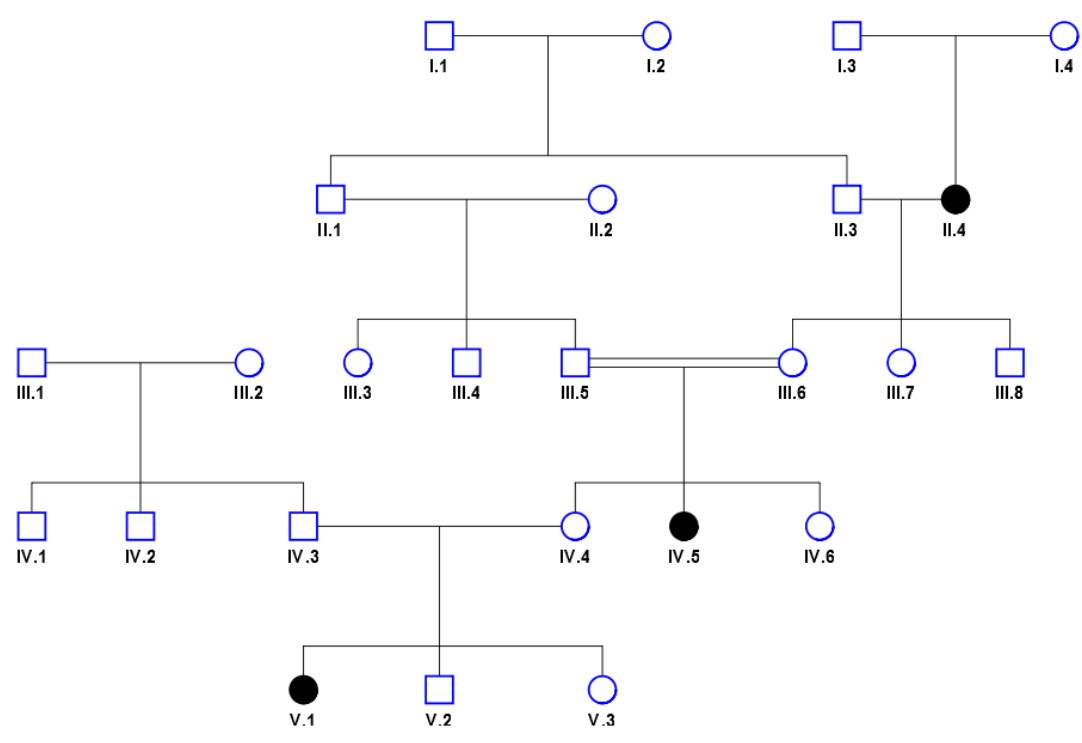

Figure 1. Family pedigree of the cases with vitiligo in three generations 
Among the various scoring system for vitiligo, we used vitiligo area scoring index (VASI), vitiligo disease activity (VIDA) and potential regimentation index (PRI) for assessment of cases (Table 1) (9).

\section{Case 1}

This patient was a 20-year-old married Persian woman. Skin depigmentation first appeared at age of 7 with emergence of $2 \mathrm{~cm}$ spots on both sides of the left ankle. Diagnosis of vitiligo was made with wood lamp examination. Medications consisting of corticosteroid topical ointment for the depigmented spots, calcium supplement and a sunscreen were immediately prescribed for the patient. The patient had been under treatment with these medicines for 4 years but no improvement was seen. Some new spots with a same size appeared on the abdominal region, neck, lips and knee. With continuing the treatment for 11 months, the new spots on the abdominal region, neck and knee were treated, but because of the lack of success in other spots and her frustration with the treatment, the treatment program was stopped. New spots continued to appear on the edges of the old ones until the age of 15, and eventually the new spots were connected to the previous depigmented spots.

New depigmented areas (1 $\mathrm{cm}$ in diameter) appeared on the nipples and fingertips. These new depigmented areas progressed rapidly, and the disease progression stopped when the spots reached size of $3 \mathrm{~cm}$ on the nipples and $2 \mathrm{~cm}$ on all fingers. At this time, the patient visited a physician again and 10 sessions of phototherapy were prescribed. Not only the results were not satisfactory, the therapy also caused side effects including skin dryness, itching, irritation and mouth sores. Therefore, the patient did not continue the treatment. No new spot was developed for 3-4 years, but the breadth of the depigmented areas on the fingertips increased and new spots appeared on the back of the hands. Still, the patient did not visit a physician (Figure 2).

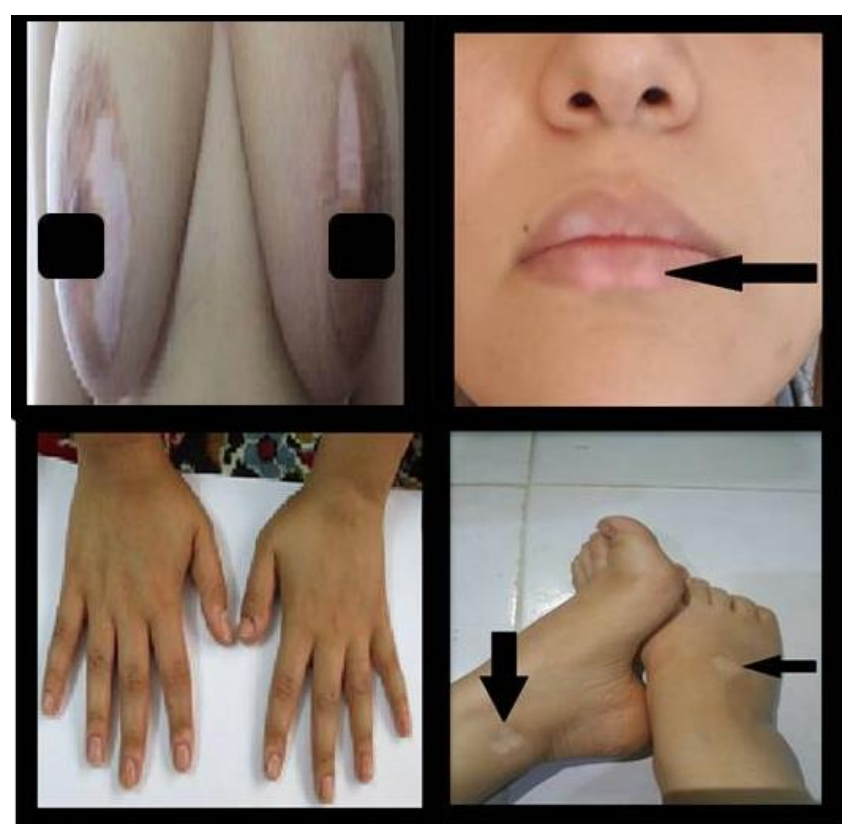

Figure 2. Presentations of the 20-year-old patient with vitiligo (case 1). Depigmentation of the skin is evident on the lip, nails, breast and foot. 


\section{Case 2}

This case was a 43-year-old Persian woman who was diagnosed with vitiligo at age of 8 by detection of $3 \mathrm{~cm}$ white spots on her chest. The initial treatment was to rub an ointment on the depigmented areas and then expose the areas to direct sunlight for 5 seconds. The patient was under the treatment until age of 17 , but the patient refused to continue the treatment due to lack of improvement. The course of the disease slowed down until age of 20 , but the spots were expanded to the breast, forearm, hands and fingertips from the age of 34 years. At the same time, numerous spots appeared on the patient's face and other areas of her body following a severe nervous shock. The new spots expanded very fast in a way that over $50 \%$ of her body became depigmented. Then, the patient once again approached a dermatologist and monobenzen ointment was prescribed for the patient. Despite using the ointment for a year, other parts of her body also became depigmented. The patient is not currently under any chemical treatment and only uses a strong sunscreen (Figure 3).

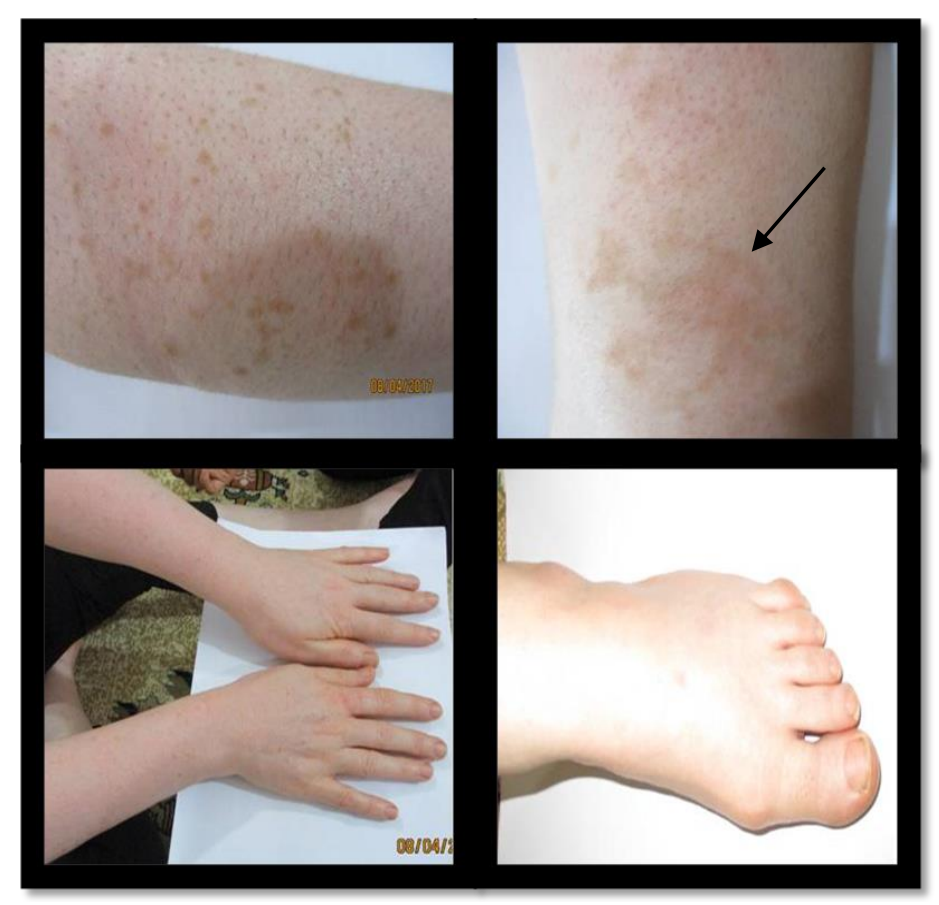

Figure 3. Presentations of the 43-year-old patient with vitiligo (case 2). Depigmentation of the skin on the feet and hands is evident.

\section{Case 3}

This case was an 85-year-old woman who was first diagnosed with vitiligo at age of 12 . The patient noticed the skin changes by observing a small, depigmented spot on her back, but never approached a doctor. At first, the disease was progressing slowly, but after an emotional stress caused by loss of relatives, the disease developed very fast, spots appeared on her face and continue to affect other areas of her body until the patient's body became completely white since 10 years ago (Figure 4 ). 


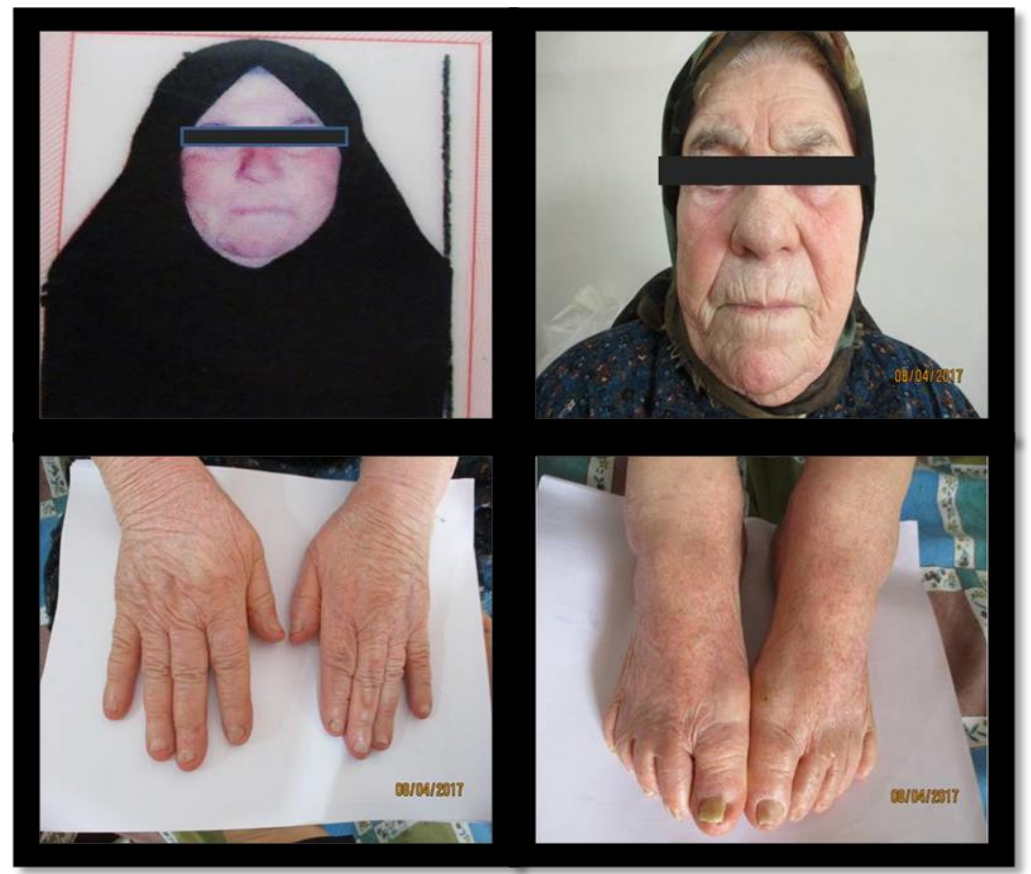

Figure 4. Presentations of the 85-year-old patient with vitiligo (case 3). The two top photos were taken at age of 60 years (left) and 85 years (right).

A brief history and characteristics of the three cases are presented in table 1.

Table 1. Clinical features, outcomes, and therapeutic and diagnostic methods of the three cases with vitiligo in one family

\begin{tabular}{|c|c|c|c|}
\hline Characteristic & Case 1 & Case2 & Case3 \\
\hline Age of onset & 7 years & 8 years & years \\
\hline Drug therapy & Yes & Yes & No \\
\hline Phototherapy & Yes & No & 85 \\
\hline Current Age & 20 & No & No \\
\hline $\begin{array}{c}\text { Cognitive } \\
\text { therapy/psychological } \\
\text { support }\end{array}$ & No & Nervous shock & Nervous shock \\
\hline Trigger & Student stress & Whole body & Whole body \\
\hline $\begin{array}{c}\text { Affected organs } \\
\text { Lips, ankles, fingertips, } \\
\text { breasts and feet }\end{array}$ & & 0 & 0 \\
\hline VIDA & -1 & $100 \%$ & $100 \%$ \\
\hline VASI & $1-2 \%$ & NA & NA \\
\hline PRI & 0 & & \\
\hline
\end{tabular}

No: no treatment, NA: not assigned 


\section{DISCUSSION}

Vitiligo is a hereditary skin disorder that equally affects men and women of different ethnicities $(10,11)$. Although different treatment programs are being used for patients with vitiligo, there is currently no cure for this disease (11). Vitiligo can be classified as localized, generalized and universal based on the amount of lesions (12). To the best of our knowledge, no study has yet evaluated incidence of vitiligo in consecutive generations of a family. According to previous studies, the prevalence of vitiligo is almost similar in men and women. However, we found that the disease only existed in three women of this family, and no man in the family was affected. Therefore, it seems that inheritance could be a key factor in the incidence of vitiligo. In this regard, a study found that 24 of 72 patients with vitiligo had a positive family history (8). The average age of onset of vitiligo has been reported to be $21.9 \pm 13.4$ years. In our cases, the age of onset was between 7 and 12 years (13).

Manolache and Petrescu-Seceleanu stated that stress is one of the most important triggering factors for vitiligo (14). In our cases, stress was also a key factor in disease progression.

The primary aim of vitiligo management is to prevent its progression. Corticosteroids with enough dosage can be effective in controlling the disease activity (15). However, the side effects of corticosteroids can be problematic. Therefore, oral mini pulse treatment with betamethasone and dexamethasone is proposed as an effective alternative for controlling the disease progression and inducing repigmentation (16). Methotrexate has been widely used as a safe and longacting drug for treatment of different autoimmune disorders, such as psoriasis, psoriatic arthritis, alopecia areata, systemic lupus erythematosus and rheumatoid arthritis (17). According to Sing et al., oral mini pulse treatment and methotrexate have similar efficacy in controlling the activity of vitiligo (18). In some cases, autologous transplantation of melanocytes is recommended after drug therapy. Narrowband ultraviolet B $(311 \mathrm{~nm})$ and monochromatic excimer light $(308 \mathrm{~nm})$ phototherapy is also considered a successful therapeutic method (12).

Topical corticosteroids are the first-line treatment for patients with segmental vitiligo. It can cause repigmentation in areas exposed to sunlight and peripheral areas in $75 \%$ and $33 \%$ of the cases, respectively. However, phototherapy could also be effective for repigmentation in $70-80 \%$ of cases, but full repigmentation can only be achieved in $20 \%$ of cases (8). Stress is a trigger factor for vitiligo (14). Silvan (19) and Papadopoulos (20) stated that $40 \%$ and $25 \%$ of vitiligo patients experienced death of a close friend/family member. Patients perceptions of etiology of vitiligo may differ, but the patients generally believe that both stress and genetic background are involved $(19,20)$. In our study, loss of a family member and stress triggered the disease and its progression in cases 2 and 3 .

\section{CONCLUSION}

Given the low therapeutic efficacy of current therapies for treatment of vitiligo and lack of a screening method, it is recommended to keep patients away from stress as much as possible. Offering genetic counseling, risk assessment and finding genes involved in the development of this disease could also be beneficial.

\section{ACKNOWLEDGMENTS}

We are deeply grateful to the family who took part in this research.

\section{DECLARATIONS \\ Funding \\ Not applicable}

\section{Ethics approvals and consent to participate}

Written consent was taken from the cases for publication of their photos.

\section{Conflict of interest}

The authors declare that there is no conflict of interest. 


\section{REFERENCES}

1. Shah R, Hunt J, Webb TL, Thompson AR. Starting to develop self-help for social anxiety associated with vitiligo: using clinical significance to measure the potential effectiveness of enhanced psychological self-help. $\mathrm{Br} \mathrm{J}$ Dermatol. 2014;171(2):332-7. [DOI:10.1111/bjd.12990]

2. Saeedinezhad F, Firouzkouhi M, Abdollahimohammad A, Rahnama M. The challenges facing with vitiligo: a phenomenological research. Int. J. Pharm. Res. Allied Sci., 2016, 5(3):356-364.

3. Aghaei S, Amiri M, Aghaei M, Nilforoushzadeh MA. Molecular genetics and epidemiology of vitiligo: a minireview. Int $\mathbf{J}$ Epidemiol Res. 2018;5(3):103-106. [DOI:10.15171/ijer.2018.22]

4. 4 .Afkhami-Ardekani M, Ghadiri-Anari A, Ebrahimzadeh-Ardakani M, Zaji N. Prevalence of vitiligo among type 2 diabetic patients in an Iranian population. Int $\mathrm{J}$ Dermatol. 2014;53(8):956-8. [DOI:10.1111/ijd.12148]

5. Saeedinezhad F, Firouzkouhi M, Abdollahimohammad A and Rahnama M , Aziz Shahrakivahed A (2017).The Lived Experiences of Persons with Vitiligo: A Phenomenological Research. JAMMR, 2017 23(9): 1-8.

6. Silverberg JI, Silverberg NB. Quality of life impairment in children and adolescents with vitiligo. Pediatr Dermatol. 2014;31(3):309-18. [DOI:10.1111/pde.12226]

7. Cupertino F, Niemeyer-Corbellini JP, RamosE-Silva M. Psychosocial effects of vitiligo. Clin Dermatol. 2017;35(3):292-297.

[DOI:10.1016/j.clindermatol.2017.01.001]

8. 8 .Spritz R, Andersen G. Genetics of Vitiligo. Dermatol Clin. 2017; 35(2): 245-255. [DOI:10.1016/j.det.2016.11.013]

9. Dicle O. Assessment Methods in Vitiligo. Pigmentary Disorders 2015; 2: 160.

10. Salah Eldin MM, Sami NA, Aly DG, Hanafy NS. Comparison Between (311-312 nm) Narrow Band Ultraviolet-B Phototherapy and (308 nm) Monochromatic Excimer Light Phototherapy in Treatment of Vitiligo: A Histopathological Study. J Lasers Med Sci. 2017; 8(3):123-127. [DOI:10.15171/jlms.2017.22]

11. Ahn Wee MD. A case report of extensive vitiligo. Hawaii Tien- Medical Journal. 1997; 56: 37-42.

12. Pacifico A, Leone G. Photo(chemo)therapy for vitiligo. Photodermatol Photoimmunol Photomed. 2011; 27(5):261-77. [DOI:10.1111/j.1600-0781.2011.00606.x]
13. Singh WG, Gomez AC, Swamy SC, Ashok $\mathrm{DN}$, and Reddy MJ. Approach to a case of vitiligo. Ind J Aerospace Med. 2009; 53(1): 62-65. 14. Manolache L, Petrescu-Seceleanu D. Stress involvement as trigger factor in different skin conditions. World J Dermatol. 2013; 2(3): 16-26. [DOI:10.5314/wjd.v2.i3.16]

15. Pasricha JS, Khaitan BK. Oral mini-pulse therapy with betamethasone in vitiligo patients having extensive or fast-spreading disease. Int $\mathbf{J}$ Dermatol. 1993;32(10):753-7. [DOI:10.1111/j.1365-4362.1993.tb02754.x] 16. Kanwar AJ, Dhar S, Dawn G. Oral minipulse therapy in vitiligo. Dermatology. 1995;190(3):251-2. [DOI:10.1159/000246705]

17. Sung JY, Hong JH, Kang HS, Choi I, Lim SD, Lee JK, Seok JH, Lee JH, Hur GM. Methotrexate suppresses the interleukin-6 induced generation of reactive oxygen species in the synoviocytes of rheumatoid arthritis. Immunopharmacology. 2000;47(1):35-44.

[DOI: $10.1016 /$ S01623109(99)00185-X]

18. Singh H, Kumaran MS, Bains A, Parsad D. A Randomized Comparative Study of Oral Corticosteroid Minipulse and Low-Dose Oral Methotrexate in the Treatment of Unstable Vitiligo. Dermatology. 2015;231(3):286-90. [DOI:10.1159/000433424]

19. Silvan M. The psychological aspects of vitiligo. Cutis 2004; 163-167.

20. Papadopoulos L, Bor R, Legg C, Hawk JL. Impact of life events on the onset of vitiligo in adults: preliminary evidence for a psychological dimension in aetiology. Clin Exp Dermatol 1998; 23: 243-248. [DOI:10.1046/j.13652230.1998.00384.x] 